Notfall Rettungsmed $2017 \cdot 20: 495-500$

DOI 10.1007/s10049-017-0312-8

Online publiziert: 4. Mai 2017

(C) Der/die Autor(en) 2017. Dieser Artikel ist

eine Open-Access-Publikation.

CrossMark

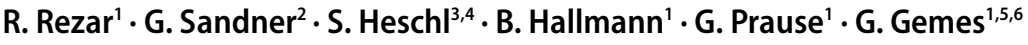 \\ ${ }^{\prime}$ Klinische Abteilung für allgemeine Anästhesiologie, Notfall- und Intensivmedizin, Universitätsklinik für \\ Anästhesiologie und Intensivmedizin, Medizinische Universität Graz, Graz, Österreich \\ ${ }^{2}$ Finanzen/IT/Telekommunikation - IKT, Österreichisches Rotes Kreuz, Graz, Österreich \\ ${ }^{3}$ Klinische Abteilung für Herz-, Thorax- und Gefäßchirugische Anästhesiologie und Intensivmedizin, \\ Universitätsklinik für Anästhesiologie und Intensivmedizin, Medizinische Universität Graz, Graz, \\ Österreich \\ ${ }^{4}$ Bergrettungsdienst Steiermark, Graz, Österreich \\ ${ }^{5}$ Abteilung für Anästhesiologie und Intensivmedizin, Krankenhaus der Barmherzigen Brüder Graz, Graz, \\ Österreich \\ ${ }^{6}$ Steirischer Flugrettungsverein, Christophorus Flugrettung, Graz, Österreich
}

\title{
Vorteile der Hubschrauberrettung am Beispiel eines österreichischen Notarzthubschraubers
}

\section{Eine retrospektive Analyse}

\section{Die Hubschrauberrettung ist in vielen Ländern ein zentrales Element der Notfallversorgung. In Österreich existiert ein dichtes Netzwerk an bodengebundenen Notarztmitteln sowie tagsüber eine flächendeckende Versorgung mit Notarzthubschraubern (NAH). Allgemein sind Notarzteinsätze mit nicht vitalbedrohten Patienten relativ häufig. Die vorliegende Arbeit evaluiert, wie oft der Einsatz eines Notarzthubschraubers gegenüber einem bodengebundenen Notarzt- oder Rettungsmittel vorteilhaft war.}

\section{Hintergrund}

Der NAH ist als schneller Notarztzubringer, Rettungsgerät im Gelände und für einen zügigen und schonenden Transport prädestiniert [7]. Durch seinen Einsatz können für kritisch kranke Patienten Überlebensvorteile entstehen $[1,2$, $5,13]$. Demgegenüber stehen erhebliche Material-, Wartungs- und Ausbildungskosten, sodass NAH prinzipiell eine eingeschränkt verfügbare Ressource darstellen. Auch wenn es sich größtenteils um
Vorhaltekosten handelt, sollte aufgrund des zunehmenden Spardrucks im Gesundheitswesen und knapper werdender Personalressourcen ein möglichst effizienter Einsatz erfolgen. Da üblicherweise $\mathrm{NAH}$ nicht redundant vorgehalten werden können, kann sich die Disposition $\mathrm{zu}$ einem weniger indizierten Fall auch nachteilig für einen anderen dringlicheren Patienten auswirken. Ob ein NAH vorteilhaft eingesetzt wird, ist eine komplexe Fragestellung. Diese Arbeit versucht der Frage nachzugehen, ob und in welchem Ausmaß die Primärrettungseinsätze eines für Österreich repräsentativen NAH gegenüber bodengebundenen Notarzt- oder Rettungsmitteln vorteilhaft waren.

\section{Methoden}

In einer retrospektiven Analyse wurden alle Einsätze des NAH Christophorus 12 (Flughafen Graz/Thalerhof) zwischen 01.07.2014 und 30.06.2015 untersucht. Der Hubschrauber vom Typ EC-135 (Airbus Helicopters) wird in öffentlichem Auftrag vom Christophorus Flugrettungsverein des Österreichischen
Automobil- und Touringclubs betrieben und durch die Rettungsleitstelle Steiermark des Österreichischen Roten Kreuzes disponiert. Das Einsatzgebiet umfasst hochalpine Regionen, ländliches Flach- und Hügelland und dicht besiedelte städtische Ballungsräume und wurde daher als für Gesamtösterreich geographisch repräsentativ angenommen. Insgesamt werden grob 900.000 Einwohner versorgt. Interhospitaltransfers sowie während des Anflugs stornierte oder nicht ausreichend dokumentierte Missionen wurden exkludiert. Wenn sich mehr als ein Patient am Notfallort befand wurde der schwerwiegendere Fall herangezogen. Für die Bewertung der Einsatzindikation wurde ein Score, bestehend aus den Kategorien Einsatzort, Notfalldiagnose und Maßnahmen erstellt, wobei in jeder Kategorie zwischen 0 und 4 Punkte vergeben wurden, sodass maximal 12 Punkte erreicht werden konnten. Die Datenauswertung erfolgte mit Microsoft ${ }^{\circledR}$ Excel unter Verwendung deskriptiv-statistischer Methoden. Metrische Daten sind als Mittelwert \pm Standardabweichung dargestellt. Diese Arbeit basiert auf einer Diplomarbeit an 
Tab. 1 Beispiele aus verwendetem Diagnosenkatalog

Keine Transportpriorität nachvollziehbar/Besserung vor Ort (OPunkte)

Besserung vor Ort/möglicher Vorteil durch Lufttransport nicht auszuschließen (1 Punkt)

Permanent ausgelenkter Vitalparameter/mittelschweres Trauma (2 Punkte)

\begin{abstract}
Schweres Trauma mit nicht auszuschließender akuter Lebensbedrohung oder möglicherweise schweren Folgeschäden (3 Punkte)

Schwerste Verletzungen/ Erkrankungen (4 Punkte)
\end{abstract} (1-2 Frakturen) Hypoglykämie rhythmus vor Ort fläche
Exitus letalis mit/ohne kardiopulmonaler Reanimation

Geschlossene Fraktur von Unterarm oder Unterschenkel

Hypertensive Entgleisung mit deutlicher Besserung vor Ort

Krampfanfall - abgelaufen oder Sistieren nach Therapie

Schmalkomplextachykardien mit Rekonversion in Sinus-

Asthmaanfall mit Besserung vor Ort

Commotio cerebri (Schädelhirntrauma erstgradig)

Transitorische ischämische Attacke - Symptomatik bereits am Einsatzort wieder vorbei

Akutes Koronarsyndrom mit persistierender Symptomatik Humerusfraktur geschlossen

Pulmonalarterienembolie (bzw. Verdacht auf eine solche)

Verbrennung Erwachsener $\geq 20 \%$, aber $<40 \%$ Körperober-

Verbrennung Kind $\geq 10 \%$, aber $<20 \%$ Körperoberfläche

Fraktur Humerus offen

Fraktur Femurschaft geschlossen

Fraktur Unterschenkel/Unterarm

geschlossen, >2 Extremitäten

Halbseitenschwäche akut

Persistierende Schocksymptomatik

Polytrauma

Reanimation primär erfolgreich

ST-Hebungsinfarkt

Wirbelsäulenverletzung mit neurologischen Ausfällen

\section{Tab. 2 Beispiele aus dem verwendeten Maßnahmenkatalog}

Keine Maßnahmen durchgeführt (OPunkte)

Wenig invasive Maßnahmen (1 Punkt)

Nur Venenzugang \pm Kristalloid $(<1000 \mathrm{ml}$ bei Erwachsenen)

Antiemetikum für den Hubschraubertransport

Paracetamol rektal

"Maßnahmenpaket leicht" (2 Punkte)

Nitropräparate, Schleifendiuretika, Urapidil bei hypertensiver Krise

Sedoanalgesie

Lebensrettende Maßnahmen/ "Maßnahmenpaket schwer" (3 Punkte)

Antiarrhythmika

Intraossärer Zugang

Continuous Positive Airway Pressure Therapie über Maske

Prähospitale Narkoseeinleitung und endotracheale Intubation

Reanimation

Invasive Maßnahmen (4 Punkte)
Entlastung eines Pneumothorax/digitale Thorakozentese Koniotomie

Zentralvenöser Zugang der Medizinischen Universität Graz, und für diese liegt ein Votum der Ethikkommission der Medizinischen Universität Graz vor (28-168 ex 15/16).

Kategorie Einsatzort. Für jeden Einsatz wurde mittels Routenserver (xServer der Fa. PTV-AG, Karlsruhe, Deutschland mit Standardeinstellungen) der kürzeste Anfahrtsweg zur geographischen Ortsmitte des Einsatzorts und die Fahrzeit des zum Einsatzort nächstgelegenen bodengebundenen Notarztmittels berechnet. Von den ermittelten Fahrzeiten wurden jeweils $20 \%$ abgezogen, um der Sondersignalfahrt Rechnung zu tragen sowie zur dokumentierten Flugzeit des NAH 2 min für Landung und Erreichen des Patienten addiert. Anschließend erfolgte der Vergleich von Fahr- vs. Flugzeit und eine Punktevergabe für den entstandenen Zeitvorteil. Einsätze auf Skipisten, Forstunfälle oder andere Fälle mit dokumentiert schwierigem Gelände wurden automatisch mit 2 Punkten, mit normalen Kraftfahrzeugen nicht erreichbare Orte oder Seilbergungen mit der maximalen Punktezahl bewertet.

Kategorie Transportpriorität. Die vor Ort gestellten Diagnosen wurden nach der daraus entstehenden Transportpriorität unterteilt, um den Vorteil eines raschen Hubschraubertransports in ein geeignetes Krankenhaus $\mathrm{zu}$ erfassen (- Tab. 1). Zur Evaluierung der diagnostischen Treffsicherheit vor Ort wurde die Diagnose am Einsatzprotokoll mit der endgültigen Diagnose laut Krankenhausinformationssystem verglichen. Dies wurde durch einen der Autoren (RR) durchgeführt; bei Unklarheiten wurde der Fall unabhängig von zwei weiteren Autoren (GG und SH) bewertet.

Kategorie Maßnahmen. Unter der Prämisse, dass vor Ort getroffene Maßnahmen die Schwere des notfallmedizinischen Zustandsbilds reflektieren, wurden vor Ort gesetzte Maßnahmen nach Invasivität gereiht in den Score aufgenommen (• Tab. 2). 


\section{Ergebnisbewertung}

Die jeweilig vergebenen Punkte aus den einzelnen Kategorieren wurden aufsummiert und das Ergebnis wie folgt bewertet (• Tab. 3):

0 Punkte. Es lag keine Vitalbedrohung vor oder es gab keine transportlogistische Begründung für einen NAH-Einsatz; dies schließt nicht aus, dass zur präklinischen Diagnosestellung ärztliches Personal erforderlich war.

1-3 Punkte. Der Fall war aufgrund notwendiger Maßnahmen vermutlich notarztpflichtig, aber ohne wesentliche Transportpriorität oder vice versa aus rettungstechnischer Sicht für den NAH geeignet. Es war jedoch weder ein zeitkritischer Transport erforderlich, noch wurde eine weitergehende ärztliche Intervention durchgeführt. Er hätte auch durch den bodengebundenen Notarztoder (Berg-)Rettungsdienst gelöst werden können.

4-12 Punkte. Die Disposition des NAH wurde als adäquat bewertet, da entweder durch die Kombination oder auch aufgrund nur eines stärker gewichteten Einzelfaktors für den Patienten ein Vorteil durch den NAH entstand. Je höher die Punkteanzahl, desto größer war theoretisch auch der entstandene Vorteil.

\section{Ergebnisse}

Im Beobachtungszeitraum wurden 1358 Einsätze registriert. Wegen unzureichender Dokumentation mussten 65 Einsätze ausgeschlossen werden, in 46 Fällen wurde der NAH bereits am Anflug storniert. Insgesamt 204 Einsätze waren Interhospitaltransfers, sodass letztlich 1043 Primäreinsätze in die Analyse einbezogen wurden ( $\bullet$ Abb. 1$)$, aus diesen der Score berechnet wurde. Bei der Ermittlung der Krankenhausdiagnose wurden ferner 121 verstorben oder lebendig am Einsatzort belassene und 73 im Krankenhausinformationssystem nicht auffindbare Patienten ausgeschlossen. So konnte von 849 Patienten die innerklinisch gestellte Diagnose ermittelt werden.

Notfall Rettungsmed 2017 $\cdot 20: 495-500 \quad$ DOI 10.1007/s10049-017-0312-8

๑ Der/die Autor(en) 2017. Dieser Artikel ist eine Open-Access-Publikation.

R. Rezar · G. Sandner · S. Heschl · B. Hallmann - G. Prause · G. Gemes

Vorteile der Hubschrauberrettung am Beispiel eines österreichischen Notarzthubschraubers. Eine retrospektive Analyse

Zusammenfassung

Hintergrund. Aufgrund der Sonderstellung von Notarzthubschraubern (NAH) ist ein effizienter Einsatz nötig. In dieser retrospektiven Arbeit wurde versucht, die Zahl adäquater Einsätze eines für Österreich repräsentativen $\mathrm{NAH}$ zu evaluieren.

Methoden. Retrospektive Datenanalyse der Primäreinsätze über 1 Jahr, Sekundäreinsätze, stornierte Einsätze oder unvollständig dokumentierte Fälle wurden ausgeschlossen. Ein Score mit den Kategorien Einsatzort, Transportpriorität und Maßnahmen wurde generiert, um den Nutzen des NAH-Einsatzes gegenüber einem bodengebundenen Notarzt- oder (Berg-)Rettungsmittel festzustellen. Anschließend erfolgte der Vergleich der Notfalldiagnose mit der Krankenhausdiagnose.
Ergebnisse. 1043 Einsätze wurden eingeschlossen. In $38 \%$ konnte der Vorteil der Hubschrauberdisposition nicht nachvollzogen werden, während in $62 \%$ die Entsendung des NAH gerechtfertigt erschien. Die vor Ort gestellten Notfalldiagnosen waren in $88 \%$ der Fälle korrekt.

Schlussfolgerung. Mehr als ein Drittel der NAH-Einsätze hätte auch mittels bodengebundenem Notarzt- oder Bergrettungsdienst gelöst werden können. Weitere Untersuchungen zur Ursachenforschung sowie Prozessund Ergebnisqualität im Flugrettungswesen sind notwendig.

Schlüsselwörter

Luftrettung · Notarzthubschrauber . Einsatzindikation · Notfalldiagnose . Qualitätskontrolle

\section{Utilization of the advantages of a physician-staffed rescue helicopter in Austria. A retrospective analysis}

\section{Abstract}

Background. Physician-staffed rescue helicopters are one of the most valuable resources in emergency medicine, so a deliberate dispatch system is of paramount importance. This retrospective study aims to determine how often the use of a helicopter was adequate at a single helicopter base in Austria.

Methods. Retrospective data evaluation, interhospital transfer missions, and missions cancelled en route were excluded. A score comprising the three categories operation site, priority of a rapid transport, and medical treatment on-scene was generated to evaluate whether the utilization of the helicopter compared to a ground-based emergency physician vehicle or mountain rescue was beneficial. For patients admitted to a hospital, the on-scene diagnosis and final diagnosis were compared.

Results. During a one-year time period,

a total of 1043 missions were included. In 38\% of the cases, no evidence of benefit of the helicopter-deployment was found, while $62 \%$ of the helicopter rescue missions were found to result in a comprehensible advantage generated by the helicopter disposition. Onscene diagnoses were found to be correct in $88 \%$ of the cases.

Conclusion. We found that more than one third of the missions attended to by a rescue helicopter could have been resolved employing ground-based emergency physician systems or mountain rescue. The reasons for this problem and further measures to ensure procedural and outcome quality in airborne emergency medical systems need to be investigated.

\section{Keywords}

Helicopter emergency medical services . Physician-staffed helicopters · Indications of dispatch · Diagnostic accuracy · Quality control 
Tab. 3 Score zur Bewertung der Einsatzindikation

\begin{tabular}{|c|c|c|c|}
\hline Punkte & Einsatzort & Transportpriorität & Maßnahmen \\
\hline 0 & Keiner nachvollziehbar & Keine Transportpriorität/Besserung vor Ort & Keine durchgeführt \\
\hline 1 & $\begin{array}{l}\mathrm{NAH} \geq 5 \text { min schneller als nächstes boden- } \\
\text { gebundenes Notarztmittel }\end{array}$ & $\begin{array}{l}\text { Besserung vor Ort/möglicher Vorteil durch Luft- } \\
\text { transport nicht auszuschließen }\end{array}$ & $\begin{array}{l}\text { Venenzugang ( } \pm \text { Antiemetikum oder } \\
\text { Kristalloid) }\end{array}$ \\
\hline 2 & $\begin{array}{l}\mathrm{NAH} \geq 10 \text { min schneller als nächstes boden- } \\
\text { gebundenes Notarztmittel }\end{array}$ & $\begin{array}{l}\text { Permanent ausgelenkter Vitalparameter/ } \\
\text { mittelschweres Trauma }\end{array}$ & "Maßnahmenpaket leicht" \\
\hline 3 & $\begin{array}{l}\text { Schwieriges Gelände, } \mathrm{NAH} \geq 15 \text { min schnel- } \\
\text { ler als nächstes bodengebundenes Notarzt- } \\
\text { mittel }\end{array}$ & $\begin{array}{l}\text { Schweres Trauma mit nicht auszuschließender } \\
\text { akuter Lebensbedrohung oder möglicherweise } \\
\text { schweren Folgeschäden }\end{array}$ & $\begin{array}{l}\text { „Maßnahmenpaket schwer", lebens- } \\
\text { rettende Maßnahme }\end{array}$ \\
\hline 4 & Seilbergung, nicht befahrbares Gebiet & Maximal zeitkritische Diagnose & Invasive Maßnahme \\
\hline
\end{tabular}

Tab. 4 Detailergebnisse Score zur Bewertung der Vorteile des Notarzthubschraubers Argument für Punktevergabe

Punkte Häufigkeit Häufigkeit absolut relativ (\%)

\section{Kategorie Einsatzort}

Keiner nachvollziehbar

NAH schnellster Zubringer $\geq 5$ min vor bodengebundenem Notarzt

$\mathrm{NAH}$ schnellster Zubringer $\geq 10$ min vor bodengebundenem Notarzt

Schwieriges Gelände, NAH schnellster Zubringer $\geq 15 \mathrm{~min}$ vor bodengebundenem Notarzt

Seilbergung/Rettung aus per PKW nicht erreichbarem Gelände

Gesamt

Kategorie Transportpriorität

Keine/Besserung vor Ort

Besserung vor Ort/möglicher Vorteil durch Lufttransport nicht auszuschließen

Permanent ausgelenkter Vitalparameter/mittelschweres Trauma

Schweres Trauma mit nicht auszuschließender akuter

Lebensbedrohung oder möglicherweise schweren Folgeschäden

Maximal zeitkritische Diagnosen

Gesamt

Kategorie Maßnahmen

Keine durchgeführt

Nur Venenzugang ( \pm Kristalloid, Antiemetika)

„Maßnahmenpaket leicht"

"Maßnahmenpaket schwer", lebensrettende Maßnahme

Invasive Maßnahme

Gesamt

Gesamtergebnisse

Keine Indikation

Für anderes Rettungsmittel geeignet

Gesamt für NAH nicht geeignet

Gesamt für NAH geeignet

Gesamt

NAH Notarzthubschrauber
Das mittlere Alter der Patienten betrug 54,5 $\pm 25,4$ Jahre, $62 \%$ der Patienten waren männlich. In $41 \%$ lagen traumatologische und in $38 \%$ internistische Notfälle vor. Insgesamt $78 \%$ der Patienten wurden durch den Hubschrauber transportiert. In $10 \%$ aller Fälle erfolgte der weitere Transfer durch bodengebundene Rettungsmittel. In $12 \%$ wurde kein Transport durchgeführt, wobei $6 \%$ aller Fälle auf erfolglose Reanimationen, $4 \%$ auf Todesfeststellungen ohne Wiederbelebungsmaßnahmen und $2 \%$ auf Fälle mit Behandlung und Verbleib vor Ort entfielen.

Bei der Evaluation des Scores zeigte sich, dass 62 \% der Einsätze mit 4 Punkten oder höher bewertet wurden und somit von adäquaten NAH-Einsätzen ausgegangen werden kann. In $38 \%$ der Fälle ergab der Score 3 Punkte oder weniger, somit konnte aus der NAH-Disposition kein Vorteil nachvollzogen werden (- Tab.4). In 36 Fällen (3\%) erfolgte in keiner Kategorie eine Punktevergabe.

Beim Vergleich der durch die NAHÄrzte gestellten Diagnosen mit den Krankenhausdiagnosen zeigte sich, dass die notärztliche Diagnose in $88 \%$ korrekt gestellt wurde. In einigen Fällen wurde das Verletzungs-/Erkrankungsausmaß unterschätzt. Hier waren v. a. falsch eingeschätzte Patienten mit schweren Thoraxtraumata ( $n=19$; Beispiel: Sternumfraktur, Pneumothorax, Serienrippenfraktur bei keinem dokumentierten Thoraxtrauma) und fehlgedeutete Pulmonalarterienembolien $(n=4$; Beispiel: vermutete Synkopen, resp. Infekt) auffällig. Bei den überbewerteten Fällen fanden sich meist Fälle von vermuteten akuten Koronarsyndromen, welchen beispiels- 


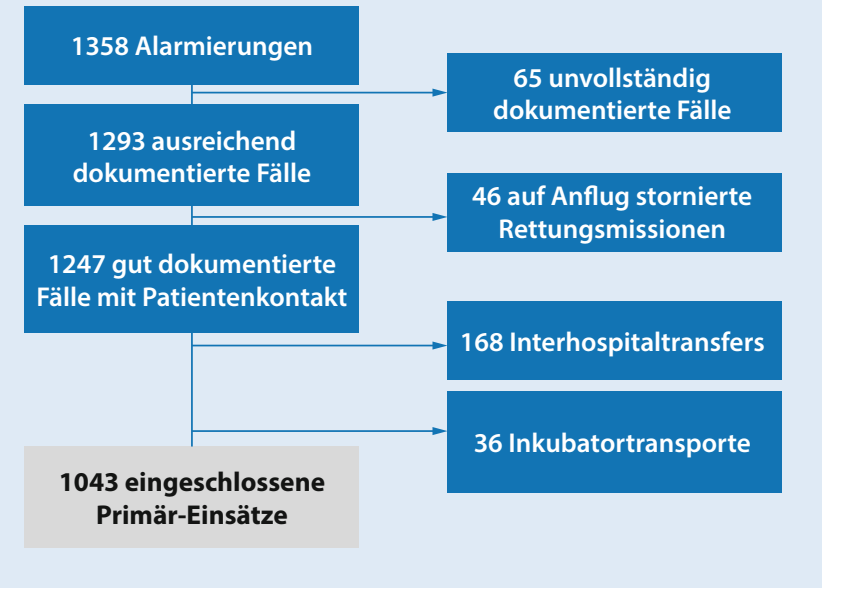

Abb. $1<$ Patientenflussdiagramm der eingeschlossenen Fälle weise respiratorische Infekte oder hypertensive Kreislaufverhältnisse zugrunde lagen. In $2 \%$ aller erfassten Einsätze wurde eine falsche Diagnose (Beispiel: Verdacht auf akutes Koronarsyndrom bei später gesicherter Choledocholithiasis als Ursache der Beschwerden oder Verdacht auf Schädelhirntrauma bei internistischer Ursache einer Synkope) gestellt.

\section{Diskussion}

Diese Untersuchung zeigt auf, dass man in einem für Österreich repräsentativen NAH-System $38 \%$ aller Einsätze vermutlich auch bodengebunden hätte lösen können. Die Disposition von Notarztmitteln wird von Faktoren wie Verfügbarkeit von Notfallmitteln, Ausbildung von Sanitätern und Leitstellendisponenten, unterschiedlichen Indikationsrichtlinien, Ausnutzung von gesetzlich vorgesehenen Notfallkompetenzen, Kostendruck und regionalen Alarmierungsgebräuchen beeinflusst [8] Zumindest in Teilen von Österreich ist die Besetzung von bodengebundenen Notarztmitteln zusehends schwieriger und der Kostendruck im Gesundheitssystem steigt stetig. Umso wichtiger erscheint es, einen NAH möglichst gezielt dort einzusetzen, wo der Benefit für den Patienten am größten erscheint. Die genauen Ursachen für die hohe Rate an Einsätzen, die auch bodengebunden hätten gelöst werden können, sind wie erwähnt komplex und sollen im Rahmen dieser Arbeit nicht evaluiert werden.
Die Vorteile eines NAH gegenüber bodengebundenen Rettungsmitteln können nicht mit einem singulären Parameter erfasst werden, weshalb diese Arbeit auf einem kombinierten Score basiert.

Während die Flugzeiten relativ genau erfasst werden konnten, sind die aus gemittelten Geodaten errechneten Anfahrtszeiten für Notarztfahrzeuge beispielsweise von Witterungsbedingungen und Verkehr beeinflussbar und daher nur Näherungswerte. Auch wurden Einsätze in denen der Hubschrauber als Rettungsgerät aus beispielsweise alpinem Gelände genutzt wurde, unabhängig von der notärztlichen Versorgung als indiziert bewertet, sodass dem Einsatz als Alpinrettungsmittel in einem Land wie Österreich Rechnung getragen wurde.

Eine wesentliche Stärke des NAH stellt der schnelle, direkte und erschütterungsfreie Transport auch über weite Strecken dar, was besonders für $\mathrm{Pa}$ tienten günstig ist, die zeitkritisch in ein Schwerpunktkrankenhaus verbracht werden müssen. Dementsprechend wurden im Score alle Diagnosen, bei denen eine verkürzte Prähospitalphase bis zur definitiven innerklinischen Versorgung einen Benefit verspricht, automatisch als für den Hubschrauber indiziert gewertet (es wurde angenommen, dass der NAH-Transport immer schneller war). Diese Sichtweise wird durch medizinische Lehrmeinungen untermauert (z. B.: „time is brain/muscle") $[4,12,14$, $15]$, während sich bei häufigen notfallmedizinischen Krankheitsbildern wie z. B.: hypertensiven Entgleisungen nach der notärztlichen Therapie vor Ort nur ein geringer prognostischer Vorteil aus einem Hubschraubertransport ergeben wird. Nicht selten kann hier sogar der Transport in einem geräumigen Fahrzeug gegenüber dem Transfer in einem engen Hubschrauber von Vorteil sein.

Die durch Flugrettungsärzte vor Ort gestellten Diagnosen wurden $\mathrm{zu} 88 \%$ von der Diagnose im Krankenhaus bestätigt, sodass die Schwere des Krankheitsbilds oder der Verletzungen und die Dringlichkeit des Transports vor Ort im Wesentlichen korrekt eingeschätzt wurden. Vor Ort gesetzte Maßnahmen lassen sicherlich auch Rückschlüsse auf die Notfallschwere zu, durch Notärzte gesetzte Maßnahmen wurden daher als dritte Kategorie in den Score aufgenommen. Vor allem bei invasiveren Maßnahmen wie Narkoseeinleitung oder digitaler Thorakozentese treten technische Fertigkeiten, Ausbildung und Erfahrung der Ärzte in den Vordergrund. Am NAH tätige Ärzte begegnen aufgrund des großen Versorgungsgebiets eines Hubschraubers schwerwiegenden Notfällen meist relativ häufig, hingegen werden für bodengebunden tätige Notärzte relativ niedrige Frequenzen bei invasiven Maßnahmen berichtet $[6,9]$. So kann der Hubschrauber oft auch als Zubringer entsprechender Spezialisten für ein großes Einsatzgebiet angesehen werden [3]; dies ist jedoch nur in Einzelfällen auch tatsächlich belegt [10].

Vor allem aus dem retrospektiven Studiendesign ergeben sich auch Limitationen dieser Arbeit. Wohl aufgrund des Zeitdrucks war die Dokumentation teilweise unvollständig, sodass knapp 5\% der Fälle ausgeschlossen werden mussten. So konnten wahrscheinlich, bedingt durch Probleme bei der Erfassung der administrativen Daten, nicht lückenlos alle Patienten in den Krankenhäusern nachverfolgt werden. Auch konnte nicht erfasst werden, ob ein bodengebundenes Notarztmittel zum gegebenen Zeitpunkt tatsächlich einsatzbereit war, sodass sich dahingehend eine deutliche Unsicherheit in Bezug auf den Zeitvorteil ergibt. Die durchschnittliche Einsatzfrequenz der bodengebundenen Notarztstützpunkte im Bundesland Steiermark beträgt jedoch 2,25 Einsätze pro 24 h pro Stütz- 
punkt [11], daher könnte dies durchaus bei einigen Einsätzen der Fall gewesen sein. Demgegenüber sei erwähnt, dass Einsätze, bei denen der NAH noch am Anflug durch andere Rettungskräfte vor Ort wieder storniert wurde, nicht in die Analyse einbezogen wurden, sodass bereits eine Vorselektion der Daten erfolgte. Grundsätzlich muss auch festgehalten werden, dass dieser Score zum ersten Mal angewandt und durchaus „aus der Praxis“ generiert wurde, jedoch nicht extern oder durch eine Fachgesellschaft validiert wurde. In den Score wurden auch keine Anforderungsdiagnosen miteinbezogen; der Score beurteilt derzeit also retrospektiv die Vor-Ort-Situation.

Insgesamt ergibt sich aus dieser Untersuchung sicherlich der Bedarf von vermehrter Evaluierung der Prozess- und Ergebnisqualität im NAH-Rettungswesen. Aus vorliegenden Daten lässt sich nicht erheben, wie viele Patienten, die klar vom NAH profitiert hätten, nur verzögert oder eventuell auch gar nicht über den Luftweg transportiert wurden, während dieser mit weniger schwerwiegenden Fällen besetzt war. Eine gewisse Anzahl an weniger indizierten Fällen ist jedoch zweifelsfrei als „Sicherheitspolster“ im Dispositionsschema einer Rettungsleitstelle notwendig; die eigentlichen Dispositionsmodalitäten sind jedoch nicht Gegenstand der Untersuchung. Aus Sicht der Autoren sollte aber aus finanziellen und einsatztaktischen Gründen versucht werden, einen NAH jenen Patienten zuzuführen, die davon am meisten profitieren können.

\section{Fazit für die Praxis}
- Die Vorteile eines NAH, nämlich schnelles Zubringen qualifizierter medizinischer Hilfe bis in entlegene Gebiete und rascher und schonender Transfer in die geeignete Zielkli- nik, wurden in einem für Österreich repräsentativen Notarzthubschrau- berstützpunkt in über einem Drittel aller Einsätze nicht ausgenutzt.
- Weitere Studien und Maßnahmen zur vermehrten Qualitätssicherung sollten durchgeführt werden, um die adäquate Disposition von Luftret-

tungsmitteln in Zukunft weiter verbessern zu können.

\section{Korrespondenzadresse}

Prim. Priv.-Doz. Dr. G. Gemes, DESA

Abteilung für Anästhesiologie und Intensivmedizin, Krankenhaus der Barmherzigen Brüder Graz Marschallgasse 12, $8020 \mathrm{Graz}$, Österreich geza.gemes@bbgraz.at

Open access funding provided by Medical University of Graz.

\section{Einhaltung ethischer Richtlinien}

Interessenkonflikt. R. Rezar, G. Sandner, S. Heschl, B. Hallmann, G. Prause und G. Gemes geben an, dass kein Interessenkonflikt besteht.

Dieser Beitrag beinhaltet keine von den Autoren durchgeführten Studien an Menschen oder Tieren.

Open Access. Dieser Artikel wird unter der Creative Commons Namensnennung 4.0 International Lizenz (http://creativecommons.org/licenses/by/4.0/deed. de) veröffentlicht, welche die Nutzung, Vervielfältigung, Bearbeitung, Verbreitung und Wiedergabe in jeglichem Medium und Format erlaubt, sofern Sie den/die ursprünglichen Autor(en) und die Quelle ordnungsgemäßnennen, einen Linkzur Creative Commons Lizenz beifügen und angeben, ob Änderungen vorgenommen wurden.

\section{Literatur}

1. Andruszkow $H$, Hildebrand $F$, Lefering $R$, Pape HC, Hoffmann R, Schweigkofler U (2014) Ten years of helicopter emergency medical services in Germany: Do we still need the helicopter rescue in multiple traumatised patients? Injury 45:53-58

2. Biewener A, Aschenbrenner U, Sauerland $S$, Zwipp H, Rammelt S, Sturm J (2005) Einfluss von Rettungsmittel und Zielklinik auf die Letalität nach Polytrauma: Eine Standortbestimmung. Unfallchirurg 108(5):370-377

3. Butler DP, Anwar I, Wilett K (2010) Is it the $\mathrm{H}$ or the EMS in HEMS that has an impact on trauma patient mortality? A systematic review of the evidence. Emerg Med J 27:692-701

4. De Luca G, Suryapranata $H$, Ottervanger JP, Antman EM (2004) Time delay to treatment and mortality in primary angioplasty for acute myocardial infarction. Every minute of delay counts. Circulation 109:1223-1235

5. Desmettre T, Yeguiayan J-M, Coadou $H$, Jacquot C, Raux M, Vivien B (2012) Impact of emergency medicalhelicopter transport directly to a university hospital trauma center on mortality of severe blunt trauma patients until discharge. Crit Care 16(5):R170

6. Gries A, Zink W, Bernhard M, Messelken M, Schlechtriemen T (2006) Realistic assessment of the physican-staffed emergency services in Germany. Anaesthesist 55:1080-1086
7. Hofer G, Voelckel WG (2014) Stellenwert der Hubschrauberrettung. Med Klin Intensivmed Notfmed 109(2):95-99

8. Prause G, Kainz J (2014) Entwicklung des Notarztwesens in Österreich. Notarzt 30:258-260

9. Prause G, Wildner G, Gemes G, Zoidl P, Zajic P, Kainz J, Pock M, Trimmel H (2017) Abgestufte präklinische Notfallversorgung - Modell Graz. Notf Rettungsmed. doi:10.1007/s10049-017-0276-8

10. Prause G, Wildner G, Kainz J, Bößner T, Gemes G, Dacar D et al (2007) Strategien zur Optimierung notärztlicher Kompetenz in der Flugrettung. Das Modell Graz. Anaesthesist 56:461-465

11. Sandner G (2015) Daten der Rettungsleitstelle des Roten Kreuzes Steiermark

12. Saver JL (2006) Time is brain - quantified. Stroke 37:263-266

13. Stewart KE, Cowan LD, Thompson DM, Sacra JC, Albrecht R (2011) Association of direct helicopter versus ground transport and in-hospital mortality in trauma patients: a propensity score analysis. Acad Emerg Med 18(11):1208-1216

14. Thomas SH, Whelan LJ, Williams E, Brown L (2013) Helicopter EMS beyond trauma: utilization of air transport for non-trauma conditions. Int JClin Med 4:511-524

15. Thomson DP, Thomas SH (2003) Guidelines for air medical dispatch. Prehosp Emerg Care 7:265-271 